\title{
Hoare's Logic Is Incomplete When It Does \\ Not Have To Be
}

\author{
J. Bergstra ${ }^{\dagger}$ \\ A. Chmielinska ${ }^{\dagger \dagger}$ \\ J. Tiuryn ${ }^{\dagger \dagger}$
}

\section{Abstract:}

If Hoare's Logic, HL( $\underline{A})$, is complete on a structure $\underline{A}$, then the set $P C(\underline{A})$ of all asserted programs true over $\underline{A}$ is recursive in the first order theory of $\underline{A}, \operatorname{Th}(\underline{A})$. We show that this implication cannot be reversed.

\section{Introduction}

It is known (cf. [5]) that for a given first order language $\ell$, the set of Floyd-Hoare partial correctness assertions $\mathrm{PC}_{\mathcal{L}}=\{\{\mathrm{P}\} \alpha\{\mathrm{Q}\} \mid \mathrm{P}, \mathrm{Q}$ are first order formulae over $\mathcal{L}, \alpha$ is a while-program over $\mathcal{L}$, and $\vDash\{P\} \alpha\{\mathrm{Q}\}$ is a $\Pi_{2}^{0}$-complete set. In particular $\mathrm{PC}_{\mathcal{L}}$ is not r.e., and therefore it cannot possess a finitary sound and (absolutely) complete proof system.

Let HL denote the standard Hoare's proof system for proving partial correctness of while-programs. Cook's [4] idea to repair incompleteness of $\mathrm{HL}$ was to take the first order

$\dagger \quad$ University of Leiden, Department of Computer Science, The Netherlands

$\dagger \dagger \quad$ University of Torun, Department of Mathematics, Poland

$\dagger \dagger$ M.I.T., Laboratory for Computer Science, USA

$t^{\dagger \dagger} \quad$ Boston University, Department of Mathematics, USA

it† University of Warsaw, Department of Mathematics, Poland.

This work was supported in part by The National Science Foundation, Grant No. MCS 8010707, and by a grant to the M.I.T. Laboratory for Computer Science by the IBM Corporation. 
theory of an $\mathcal{L}$ - structure $\underline{A}$, abbreviated Th( $\underline{A})$, as additional axioms, obtaining in this way an enlarged proof system $\mathrm{HL}(\underline{\mathrm{A}})$. In general for a given $\mathcal{L}$-structure $\underline{\mathrm{A}}$, the set $\mathrm{PC}(\underline{A})=\{\{\mathrm{P}\} \alpha\{\mathrm{Q}\} \mid \mathrm{A} \vDash\{\mathrm{P}\} \alpha\{\mathrm{Q}\}\}$ is $\Pi_{1}^{0}$ in $\mathrm{Th}(\underline{\mathrm{A}})$ (cf. [1]), and hence still is not r.e. even in $T h(\underline{A})$. Thus $H L(\underline{A})$ cannot be complete for all $\underline{A}$ 's.

For some structures $\underline{A}$, however, $\operatorname{HL}(\underline{A})$ is complete. Cook's theorem [4] says that for every structure $\underline{A}$ for which $\mathcal{L}$ is expressive for while-programs, $H L(\underline{A})$ is complete. However, expressiveness is not necessary for completeness of the HL system: any non standard model of arithmetic possess a complete $\mathrm{HL}$, but the first order language of arithmetic is never expressive for while-programs over such model (cf. [2]). Moreover, expressiveness represents what amounts to a degenerate case: when expressiveness holds, programming logic (e.g. regular Dynamic Logic) collapses to first order logic, and in particular partial correctness assertions reduce to first order formulae.

From the above it follows that if for a given structure $\underline{A}, \mathrm{HL}(\underline{A})$ is complete, then $\mathrm{PC}(\underline{A})$ is recursive in $T h(\underline{A})$. This paper is motivated by the following question: is $H L(\underline{A})$ complete for every structure $\underline{A}$ such that $\mathrm{PC}(\underline{A})$ is recursive in $\operatorname{Th}(\underline{A})$ ? It is our aim to show that the answer to the above question is negative. We will present a general construction of counterexamples for this situation. As a corollary of our results we obtain an example of a structure which shows that ability of coding finite sequences cannot be removed from assumptions of Harel's theorem on arithmetic universes (cf. [5]).

Suppose now for a while that $H L(\underline{A})$ is incomplete but $P C(\underline{A})$ is recursive in $\operatorname{Th}(\underline{A})$. What can be done to make HL complete? For this question it is meaningful to consider $H L(L, E)$ as a proof system over a first order theory $E$ in language $\mathcal{L} . \mathrm{HL}(\underline{A})$ may be then identified with $\mathrm{HL}(\ell, T h(\underline{A}))$, where $\underline{A}$ is an $\ell$-structure. It follows from [3] that $\underline{A}$ can be expanded to an $\ell^{*}$-structure $\underline{A}^{*}$ with $\ell^{*}-\ell$ being finite, such that for some decidable theory $T \subseteq \operatorname{Th}\left(\underline{A}^{*}\right)$, $P C(\underline{A}) \subseteq H L\left(\ell^{*}, T h(\underline{A}) \cup T\right)$. Thus $T h(\underline{A}) \cup T$, where $T$ is decidable but formulated in an extended language contains enough information to derive all of $\mathrm{PC}(\underline{A})$. 


\section{Preliminaries}

1.1 For completeness sale we start with a formulation of $\mathrm{HL}$ that suits our purposes best. There are four rules of inference.

\subsubsection{Assignment rule}

$$
\begin{gathered}
P \Rightarrow Q[t / x] \\
\{P\} x:=t\{Q\}
\end{gathered}
$$

where $P, Q$ are first order formulae, $x$ is a variable, $t$ is a term, and $Q[t / x]$ denotes a formula obtained from $Q$ by uniformly substituting $t$ for all free occurences of $x$ in $Q$.

\subsubsection{Composition rule}

$$
\frac{\{\mathrm{P}\} \alpha\{\mathrm{Q}\},\{\mathrm{Q}\} \beta\{\mathrm{R}\}}{\{\mathrm{P}\} \alpha ; \beta\{\mathrm{R}\}}
$$

where $\mathrm{P}, \mathrm{Q}, \mathrm{R}$ are first order formulae, and $\alpha, \beta$ are while-programs.

\subsubsection{Conditional rule}

$$
\{\mathrm{P} \wedge \mathrm{B}\} \alpha\{\mathrm{Q}\},\{\mathrm{P} \wedge \neg \mathrm{B}\} \beta\{\mathrm{Q}\}
$$

$\{\mathrm{P}\}$ if $\mathrm{B}$ then $\alpha$ else $\beta$ fi $\{\mathrm{Q}\}$

where $\mathrm{P}, \mathrm{Q}, \mathrm{B}$ are first order formulae, $\mathrm{B}$ is quantifier free, and $\alpha, \beta$ are while-programs.

\subsubsection{Iteration rule}

$$
\underline{P \Rightarrow R},\{R \wedge B\} \alpha\{R\}, R \wedge \neg B \Rightarrow Q
$$

$\{\mathrm{P}\}$ while $\mathrm{B}$ do $\alpha$ od $\{\mathrm{Q}\}$

where $P, R, Q, B$ are first order formulae, $B$ is quantifier free, and $\alpha$ is a while-program.

\subsubsection{Oracle axiom (for a given structure $\underline{A}$ ). Every $P \in T h(\underline{A})$ is an axiom.}

Given a structure $\underline{A}, \mathrm{HL}(\underline{\mathrm{A}})$ denotes the set of all asserted programs $\{\mathrm{P}\} \alpha\{\mathrm{Q}\}$ provable by using rules $(1.1 .1 \cdot 1.1 .4)$ and oracle axiom for $\underline{A}$. 
The reader can easily check that the following rule

\section{Consequence rule}

$$
P \Rightarrow P_{1},\left\{P_{1}\right\} \alpha\left\{Q_{1}\right\}, Q_{1} \Rightarrow Q
$$

$\{\mathrm{P}\} \alpha\{\mathrm{Q}\}$

where $P, P_{1}, Q, Q_{1}$ are first order formulae, and $\alpha$ is a while-program; is derivable in $H L$.

Another rule easily derivable in $\mathrm{HL}$ is the following:

$$
\frac{\{P\} \propto\{Q\}}{\{\exists \times P\} \propto\{\exists \times Q\}}
$$

where $\mathrm{P}, \mathrm{Q}$ are first order formulae, $\alpha$ is a while-program and $\mathrm{x}$ does not occur in $\alpha$.

We will need a slightly stronger statement. Let a proof of $\{\mathrm{P}\} \alpha\{\mathrm{Q}\}$ in HL.(A) be given and let $\mathrm{x}$ does not occur in $\alpha$. This proof can be transforwarded into another one by putting $\{\exists \times P\} \propto\{\exists \times Q\}$. Repeated application of this procedure yields the following lemma.

\subsubsection{Lemma}

Let $X$ be the set of all variables occuring free in $P, Q$, or $\alpha$. If $H L(A)$ proves $\{P\} \alpha\{Q\}$, then there exists a proof of $\{P\} \alpha\{Q\}$ in $H L(\underline{A})$ using only invariants and intermediate assertions with free variables in $\mathrm{X}$.

1.2 As the next step in this technical introduction we will explain a construction of a structure $\underline{A} \oplus \underline{B}$ on which our paper is technically based.

Let $L_{1}$, and $\ell_{2}$ be two disjoint similarity types. Let $A, B, \perp$ be new symbols not belong to $\mathcal{L}_{1} \cup \ell_{2}$. $A$ and $B$ are unavy predicate symbols, and $\perp$ is a constant symbol. Let $\underline{A}, \underline{B}$ be $\ell_{1}-, \ell_{2}$-structures, respectively. Assume that carriers of $\underline{A}$ and $\underline{B}$ (denoted by $|\underline{A}|$ and $|\underline{B}|$, respectively) are disjoint. Let $\mathcal{L}=\mathcal{L}_{1} \cup \mathcal{L}_{2} \cup\{A, B, \perp\} . \underline{A} \oplus B$ is an $\mathcal{L}$-siructure with carrier $|\underline{A} \oplus \underline{B}|=|\underline{A}| \cup|\underline{B}| \cup\{\perp\}$, where $\perp$ is a new element, not in $|\underline{A}| \cup|\underline{B}| . \quad A$ is a characteristic predicate of $|\underline{A}|$, and $B$ is a characteristic predicate of $|\underline{B}| . \quad L_{1}$ (respectively $L_{2}$ ) function symbols are defined the same as in $\underline{A}$ (as in $\underline{B}$, respectively), provided all arguments are taken from $|\underline{A}|$ (from $|\underline{B}|$, resp.) getting value $\perp$ otherwise. $\ell_{1}$ (respectively $\ell_{2}$ ) predicate 
symbols are defined the same as in $\underline{A}$ (as in $\underline{B}$, resp.), provided all arguments are taken from $|\underline{A}|$ (from $|\underline{B}|$, resp.), and set to be false elsewhere.

$\underline{A} \oplus \underline{B}$ is a kind of disjoint union of $\underline{A}$ and $\underline{B}$. Clearly a meaningful alternative to this definition would be a two-sorted structure. We will not do so mainly with the purpose in mind to keep as close as possible to the standard Hoare formalism.

We are now in position to formulate a general theorem which has the claim in the introduction as a corollary.

\subsubsection{Theorem}

(i) For every $\underline{A}$ there is a structure $\underline{B}$ such that $\mathrm{PC}(\underline{A} \oplus \underline{B})$ is recursive in $\operatorname{Th}(\underline{A} \oplus \underline{B})$.

(ii) For arbitrary $\underline{A}$ and $\underline{B}$, if $H L(\underline{A})$ is incomplete, then so is $H L(\underline{A} \oplus \underline{B})$.

It follows from the above result that if we take $\underline{A}$ such that $H L(\underline{A})$ is incomplete (e.g. $\underline{A}$ can be chosen to be $\langle\omega, \mathrm{s}, 0\rangle$, where $\mathrm{s}$ is a successor function on nonnegative integers, or $\underline{A}$ can be the field of complex numbers, cf. [1] for proofs of incompleteness of $H L(\underline{A})$ for these $\underline{A}$ 's), and choose $\underline{B}$ such that $\operatorname{PC}(\underline{A} \oplus \underline{B})$ is recursive in $\operatorname{Th}(\underline{A} \oplus \underline{B})$, then $H L(\underline{A} \oplus \underline{B})$ is also incomplete, and $\underline{A} \oplus \underline{B}$ is the kind of structure we looked for.

Let $\underline{N}$ stand for the standard model of arithmetic. By 1.2.1(ii) we have that for any $\underline{A}$ with incomplete $H L(\underline{A}), H L(\underline{A} \oplus \underline{N})$ is incomplete. Harel's theorem on arithmetical universes (cf. [5]) says that if $\underline{B}$ is a structure which contains the standard model of arithmetic (as a first order definable part of $\underline{B}$ ) and if $\underline{B}$ has "ability" to code finite sequences of $\epsilon$ lements from $|\underline{B}|$, then the first order language is expressive for while-programs over $\underline{B}$, and therefore $H L(\underline{B})$ is complete. Since obviously $\underline{N}$ is a first order definable part of $\underline{A} \oplus \underline{N}$, this shows that quite technical assumption of being able to code finite sequences cannot be removed from Harel's theorem.

The proofs of (i) and (ii) are quite independent and are subjects of sections 2 and 3. 


\section{Adding an expressive structure (proof of (i))}

Let $\underline{A}$ be a structure of signature $L_{1}$. If $\underline{A}$ is finite, then choose any finite $\underline{B}$. In that case $\underline{A} \oplus \underline{B}$ is finite as well and $P C(\underline{A} \oplus \underline{B})$. is recursive in $T h(\underline{A} \oplus \underline{B})$. This rerifies theorem 1.2.1(i) in that case.

Assume now that $\underline{A}$ is infinite. $\underline{B}$ is constructed as follows. Let $\underline{A}^{*}$ be an isomorphic copy of $\underline{A}$ such that $|\underline{A}| \cap\left|\underline{A}^{*}\right|=\varnothing$. Let $\mathcal{L}_{1}{ }^{*}$ be a similarity type of $\underline{A}^{*}$, such that $\ell_{1}{ }^{*}$ is an isomorphic copy of $\ell_{1}$ and disjoint with $\ell_{1}$. Then we expand $\underline{A}^{*}$ to an arithmetic universe in the sense of [5] (i.e. we add a definning predicate for "non negative integers" $N$, arithmetic operations, a relation for coding finite sequences, and in addition, for technical reasons, we add pairing and unpairing functions). We also expand the language of $\underline{A}^{*}$ by three new constants $\underline{a}, \underline{b}, \underline{\perp}$ denoting different elements. The resulting structure is $\underline{B}$. It has the same domain as $\underline{A}^{*}$, but it has got a richer similarity type which we will denote by $\ell_{2}$.

It is clear that

2.1 $\operatorname{Th}(\underline{B})$ is recursive in $\operatorname{Th}(\underline{A} \oplus \underline{B})$.

It follows immediately from the definition of $\oplus$-construction.

Because $\underline{B}$ is expressive (being an arithmetical universe), $\mathrm{HL}(\underline{B})$ is complete. Therefore

2.2 $\mathrm{PC}(\underline{B})$ is recursive in $\operatorname{Th}(\underline{B})$. By (2.1) and (2.2) it is enough to prove that

$2.3 P C(\underline{A} \oplus \underline{B})$ is many-one reducible to $P C(\underline{B})$.

We will outline the proof of (2.3) by showing an effective simulation of computations on $\underline{A} \oplus \underline{B}$ by those on $\underline{B}$. The details of this simulation are slightly messy but completely harmless and we take the liberty to skip some of them.

Let $M:|\underline{A}| \Rightarrow|\underline{B}|$ be a bijective mapping which corresponds to the isomorphism $\underline{A} \simeq \underline{A}^{*}$. First we code $\underline{A} \oplus \underline{B}$ in $\underline{B} \times \underline{B}$ as follows:

$$
\begin{aligned}
\operatorname{Code}(x)= & (\underline{a}, M(x)), \quad \text { if } x \in|\underline{A}| \\
& (\underline{\perp}, \underline{\perp}), \quad \text { if } x \in|\underline{B}|
\end{aligned}
$$


In order to describe a smooth translation of assertions and programs we introduce two infinite families of new variables: $y_{0}, y_{1}, \ldots, z_{0}, z_{1}, \ldots$

As a next step we show an effective translation $\operatorname{Tr}$ of first order formulae over the language of $\underline{A} \oplus \underline{B}$ which use individual variables: $x_{0}, x_{1}, \ldots$ to first order formulae over $\ell_{2}$ with variables $y_{0}, y_{1}, \ldots, z_{0}, z_{1}, \ldots$. The translation will have the following property: for every $P\left(x_{0}, \ldots, x_{n \cdot 1}\right)$ over the language of $\underline{A} \oplus \underline{B}$, and for all $c_{0}, \ldots, c_{n-1} \in|\underline{A} \oplus \underline{B}|$, $\underline{A} \oplus \underline{B} \vDash P\left(x_{0}, \ldots, x_{n-1}\right)\left[c_{0}, \ldots, C_{n-1}\right]$ iff $\underline{B} \vDash \operatorname{tr}(P)\left(y_{0}, z_{0}, \ldots, y_{n-1}, z_{n-1}\right)\left[\operatorname{code}\left(c_{0}\right), \ldots, \operatorname{code}\left(c_{n-1}\right)\right]$.

Because the formal definition of $\mathrm{Tr}$ is slightly cumbersome we present its details. We first introduce some notations. For a term $t$ and for $i \in\{1,2\}, \mathcal{L}_{i}(t)$ is a truth value equal to true iff $t$ is a term over language $\ell_{i}$.

Moreover, if $t$ is a term over $\mathcal{L}_{1}$, then $\tilde{f}$ denotes the corresponding term over $\mathcal{L}_{1}{ }^{*} \subseteq \mathcal{L}_{2}$.

We define $T r$ inductively. Let $P$ be $t=t^{\prime}$, where $t$ contains the variables $X=\left\{x_{i_{1}}, \ldots, x_{i_{k}}\right\}$, and $t^{\prime}$ contains the variables $X^{\prime}=\left\{x_{j_{4}}, \ldots, x_{j_{m}}\right\}$. Then $\operatorname{Tr}(P)$ is

$2.4 \quad$ "t in $\underline{A}$, and $t^{\prime}$ in $\underline{A}$, and $t=t^{\prime} " \quad V$

2.5 "t in $\underline{B}$, and $t^{\prime}$ in $\underline{B}$, and $t=t^{\prime} " \quad V$

2.6 " $t$ is $\perp$, and t' is $\perp$ "

Fomulae (2.4) - (2.6) can formally be written as follows:

$$
\begin{aligned}
& \bigwedge_{x_{i} \in X \cup X^{\prime}}\left(y_{i}=\underline{a}\right) \wedge L_{1}(t) \wedge L_{2}\left(t^{\prime}\right) \wedge \\
& \tilde{t}\left[z_{i_{1}} / x_{i_{1}}, \ldots, z_{i_{k}} / x_{i_{k}}\right]=\tilde{t}\left[z_{j_{1}} / x_{j_{1}}, \ldots, z_{i_{m}} / x_{j_{m}}\right] .
\end{aligned}
$$

$$
\begin{aligned}
& \bigwedge_{x_{i} \in X \cup X^{\prime}}\left(y_{i}=\underline{b}\right) \wedge \ell_{2}(t) \wedge \ell_{2}\left(t^{\prime}\right) \wedge \\
& t\left[z_{i_{1}} / x_{i_{1}}, \ldots, z_{i_{k}} / x_{i_{k}}\right]=t^{\prime}\left[z_{j_{1}} / x_{j_{1}}, \ldots, z_{j_{m}} / x_{j_{m}}\right] \\
& {\left[x_{x_{i}} \in X\left(y_{i}=\perp\right) \vee \quad\left(\neg \ell_{1}(t) \wedge \neg \ell_{2}(t)\right) \vee\right.}
\end{aligned}
$$

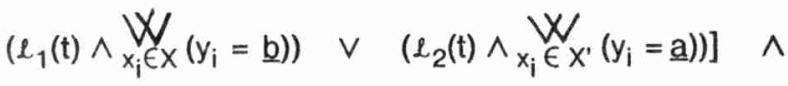




$$
\begin{aligned}
& \underset{\left[x_{i} \in X^{\prime} y_{1}=\perp \vee\right.}{W}\left(\neg \ell_{1}\left(t^{\prime}\right) \wedge \neg \ell_{2}\left(t^{\prime}\right)\right) \vee \\
& \left.\left(L_{1}\left(t^{\prime}\right) \wedge \underset{x_{i} \in X^{\prime}}{W}\left(y_{i}=\underline{b}\right)\right) \quad \vee \quad\left(\ell_{2}\left(t^{\prime}\right) \wedge \underset{x_{i} \in X^{\prime}}{W}\left(y_{i}=\underline{a}\right)\right)\right] .
\end{aligned}
$$

If $P$ is $R\left(t_{0}, \ldots, t_{n-1}\right)$ with $R \in \ell_{1}$ and $X=\left\{x_{i_{1}}, \ldots, x_{i_{k}}\right\}$ are all variables occuring in $P$ then $\operatorname{Tr}(P)$ is:

$$
\begin{aligned}
& \bigwedge_{x_{i} \in X}\left(y_{i}=\underline{a}\right) \wedge{\bigwedge_{j=0}^{n-1} L_{1}\left(t_{j}\right) \wedge}_{\sim} \\
& \tilde{R}\left(\tilde{t}_{0}\left(z_{i_{1}} / x_{i_{1}}, \ldots, z_{i_{k}} / x_{i_{k}}\right), \ldots, \tilde{t}_{n-1}\left(z_{i_{k}} / x_{i_{k}}, \ldots, z_{i_{k}} / x_{i_{k}}\right)\right) \text {., where } \tilde{R} \in \mathcal{L}_{1}{ }^{*} \text { is a } \\
& \text { symbol which corresponds to } \mathrm{R} \text {. }
\end{aligned}
$$

If $P$ is $A(t)$, then $\operatorname{Tr}(P)$ is:

$$
\mathbb{X}_{x_{i} \in X}\left(y_{i}=\underline{a}\right) \wedge \quad L_{1}(t) .
$$

The cases $P$ is $B(t)$ or $P$ is $R\left(t_{0}, \ldots, t_{n-1}\right)$ with $R \in \ell_{2}$ are dealt similarly and are left for the reader.

If $P$ is $P_{1} \vee P_{2}$, then $\operatorname{Tr}(P)$ is $\operatorname{Tr}\left(P_{1}\right) \vee \operatorname{Tr}\left(P_{2}\right)$.

If $P$ has free variables $X=\left\{x_{i_{1}}, \ldots, x_{i_{m}}\right\}$, then $\operatorname{Tr}(\neg P)$ is

$$
\bigwedge_{x_{i} \in X}\left(y_{i}=\underline{a} \vee y_{i}=\underline{b} \quad \vee \quad y_{i}=\perp\right) \wedge \neg \operatorname{Tr}(P) .
$$

And finally $\operatorname{Tr}\left(\exists x_{i} P\right)$ is

$$
\exists y_{i} \exists z_{i}\left(\left(y_{i}=\underline{a} \vee y_{i}=\underline{b} \quad \vee \quad y_{i}=\underline{\perp}\right) \wedge \operatorname{Tr}(P)\right) .
$$

This describes $\operatorname{Tr}$ in detail.

The next step is to extend $\operatorname{Tr}$ to programs $\alpha$ over the language of $\underline{A} \oplus \underline{B}$ in order to have this property for all first order formulae $P, Q$ over the language of $\underline{A} \oplus \underline{B}$ :

\section{$2.7 \underline{A} \oplus \underline{B} \vDash\{P\} \alpha\{Q\} \quad$ iff $\underline{B} \vDash\{\operatorname{Tr}(P)\} \operatorname{Tr}(\alpha)\{\operatorname{Tr}(Q)\}$.}

Given a program $\alpha$ and let $\left\{\mathrm{x}_{0}, \ldots, \mathrm{x}_{\mathrm{n} \cdot 1}\right\}$ be all variables occuring in $\alpha . \operatorname{Tr}(\alpha)$ will be using variables $y_{0}, z_{0}, \ldots, y_{n-1}, z_{n-1}$ in such a way that the following diagram commutes 


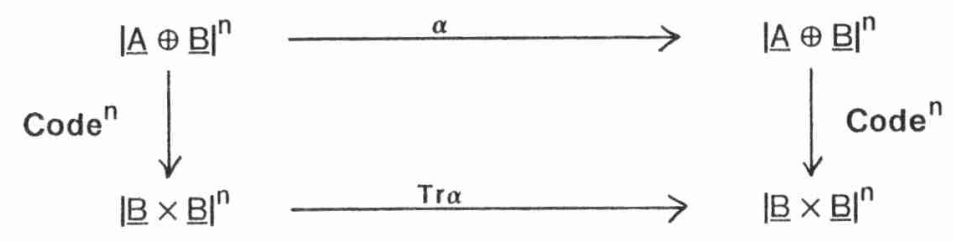

To achieve this every assignment statement $x_{i}:=t$ in $\alpha$ is replaced by an assignment statement to both $y_{i}$ and $z_{i}$, depending on $\ell_{1}(t), \ell_{2}(t)$, and the values of $y_{j}$ 's corresponding to $x_{j}$ 's occuring in t. Every test $P$ in $\alpha$ is replaced by $\operatorname{Tr}(P)$. The details of this construction can safely be left for the reader.

By (2.7), $\mathrm{PC}(\underline{A} \oplus \underline{B})$ is many-one reducible to $\mathrm{PC}(\underline{B})$. This completes the proof of part (i) of Theorem 1.2.1. 


\section{Hoare's Logic over $\underline{A} \oplus \underline{B}$ (Proof of (ii))}

In this section we will show that incompleteness of $H L(\underline{A})$ implies incompleteness of $H L(\underline{A} \oplus \underline{B})$.

First we introduce some notations. Let $\mathrm{P}$ be a first order formula over the language of $\underline{A} \oplus \underline{B} .(P)_{A}$, a relativization of $P$ to $|\underline{A}|$ is defined inductively as follows:

$$
\begin{aligned}
& \text { if } P \text { is atomic, then }(P)_{A} \text { is } P \\
& (\neg P)_{A} \text { is } \neg(P)_{A} \\
& \left(P_{1} \vee P_{2}\right)_{A} \text { is }\left(P_{1}\right)_{A} \vee\left(P_{2}\right)_{A} \\
& (\exists x P)_{A} \text { is } \exists x\left(A(x) \wedge(P)_{A}\right) .
\end{aligned}
$$

If $X$ is a finite set of variables, then $A(X)$ will always denote $\underset{X \in X}{X} A(x) .(P)_{B}$ and $B(X)$ are defined similarily.

We can now formulate on important lemma connecting $\mathrm{PC}(\mathrm{A})$ and $\mathrm{PC}(\underline{\mathrm{A}} \oplus \underline{\mathrm{B}})$.

\section{$3.1 \quad$ Lemma}

Let $P, Q$ be first order formulae over $\mathcal{L}_{1}$, and let $\alpha$ be a while-program over $\mathcal{L}_{1}$. Let $X$ be the set of all variables occuring free in $\mathrm{P}$ or $\mathrm{Q}$ or $\alpha$.

(i) $\quad \underline{A} \vDash\{P\} \alpha\{Q\} \quad$ iff $\underline{A} \oplus \underline{B} \vDash\left\{A(X) \wedge(P)_{A}\right\} \alpha\left\{A(X) \wedge(Q)_{A}\right\}$.

(ii) If $H L(\underline{A} \oplus \underline{B})$ proves $\left\{A(X) \wedge(P)_{A}\right\} \alpha\left\{A(x) \wedge(Q)_{A}\right\}$ using only invariants and inter-mediate assertions with free variables in $X$ and of the form $A(X) \wedge(R)_{A}$, then $H L(A)$ proves $\{P\} \alpha\{Q\}$.

Both parts of the above lemma are obvious and the proof is omitted. The result at this stage can be proved using the following proposition.

\subsection{Proposition}

Let $P$ be a first order formula over the language of $\underline{A} \oplus \underline{B}$. Let $X$ be the set of all variables occuring free in $\mathrm{P}$. there exists a first order formula $\mathrm{Q}$ over $\mathcal{L}_{1}$ such that

$$
\underline{A} \oplus \underline{B} \vDash(A(X) \wedge P) \leftrightarrow\left(A(X) \wedge(Q)_{A}\right) .
$$


Before we start proving Proposition 3.2 we show how to complete the proof of Theorem 1.2.1. Assume that $H L(\underline{A})$ is incomplete. Choose $\{P\} \alpha\{Q\}$ true in $\underline{A}$ but not derivable in $H L(A)$. Let $X$ be the set of all variables occuring free in $P, Q$, or $\alpha$. By Lemma 3.1.(i), $\left\{A(X) \wedge(P)_{A}\right\} \alpha\left\{A(X) \wedge(Q)_{A}\right\}$ is true in $\underline{A} \oplus \underline{B}$, and therefore if $H L(\underline{A} \oplus \underline{B}) \vdash\left\{A(X) \wedge(P)_{A}\right\} \alpha$ $\left\{A(X) \wedge(Q)_{A}\right\}$.

We derive a contradiction from this fact. Choose a proof of this asserted program in $H L(\underline{A} \oplus \underline{B})$ and use Lemma 1.1.1 to transform it into a proof in which all intermediate assertions and invariants have their free variables in $X$. Conjuncting $A(X)$ to all of these will not disburb the proof. Then, using Proposition 3.2, all invariants and intermediate assertions can be written in the form $A(X) \wedge(R)_{A}$ with $R$ a first order formula over $\ell_{1}$. By Lemma 3.1(ii), $\mathrm{HL}(\underline{A})$ proves $\{\mathrm{P}\} \alpha\{\mathrm{Q}\}$ in contrast to our assumptions.

The proof of Proposition 3.2 follows from a series of auxiliary lemmas stated below.

\subsection{Lemma $(\perp$ - elimination)}

For every first order formula $P$ over $\ell_{1} \cup\{A, B, \perp\}$ there is a formula $P^{\perp}$ over $\ell_{1} \cup\{A$, B] such that

$$
\begin{aligned}
& \text { (i) } \quad \underline{A} \oplus \underline{B} \vDash(P) \leftrightarrow P^{\perp}
\end{aligned}
$$

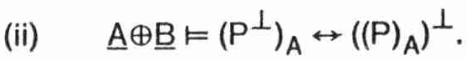

Proof: The proof is by induction on structure of $P$. The basis step is long due to a large number of cases to be considered. We show only one - the other cases for the basis step are dealt similarly.

Let $P$ be $t_{1}=t_{2}$ where $t_{1}, t_{2}$ are terms, and assume that $\perp$ occurs in $t_{1}$. It is easy to see that over $\underline{A} \oplus \underline{B} t_{1}$ assumes constantly value $\perp$. Consider the following three sub-cases for $t_{2}$ :

3.3.1 $\perp$ occurs in $t_{2}$

3.3.2 $t_{2}$ is a variable, say $x$

3.3.3 remaining cases. 
For (3.3.1) we define $\mathrm{P}^{\perp}$ as true. For (3.3.2) $\mathrm{P}^{\perp}$ is $\neg \mathrm{A}(\mathrm{x}) \wedge \neg \mathrm{B}(\mathrm{x})$. Finally for (3.3.3), if $\mathrm{X}$ is the set of all variables occuring in $t_{2}$, then we set $P^{\perp}$ to be $\neg A(X)$, i.e. $x_{i} \in X \neg A\left(x_{i}\right)$. Observe that if $X=\varnothing$ then $P^{\perp}$ becomes false. It is easy to check that $P^{\perp}$ defined above satisfies (i) and (ii). The inductive step is straightforward.

$$
\begin{aligned}
& (P \wedge Q)^{\perp} \text { is } P^{\perp} \wedge Q^{\perp} \\
& (\neg P)^{\perp} \text { is } \neg\left(P^{\perp}\right) \\
& (\forall \times P)^{\perp} \text { is } \quad \forall \times\left(P^{\perp}\right) .
\end{aligned}
$$

Again it is very easy to check that $\mathrm{P}^{\perp}$ has the desired properties.

\subsection{Lemma}

Let $P$ be a first order formula over $\mathcal{L}_{1} \cup\{A, B\}$, and assume that $x$ occurs free in $P$. There exists a formula $P^{(x)}$ over $\ell_{1} \cup\{A, B\}$ such that $x$ is not free in $P^{(x)}$, and

(i) $\underline{A} \oplus \underline{B} \vDash B(x) \Rightarrow\left(P \leftrightarrow P^{(x)}\right)$

(ii) $\underline{A} \oplus \underline{B} \vDash B(x) \Rightarrow\left\{\left(P^{(x)}\right)_{A} \leftrightarrow\left((P)_{A}\right)^{(x)}\right\}$.

Proof. The proof is by induction on P. Again, the basis step is quite long due to a large number of cases to be considered, and as in the previous proof we will discuss here only one case in detail leaving for the reader the remaining one.

Suppose $P$ is $t_{1}=t_{2}$ and consider the following possibilities.

\subsubsection{Both $t_{1}$ and $t_{2}$ are $x$}

3.4.2 $x$ occurs in $t_{1}$ and in $t_{2}$ and neither $t_{1}$ nor $t_{2}$ is $x$

3.4.3 $t_{1}$ is $x, t_{2}$ is not $x$ and $x$ occurs in $t_{2}$

3.4.4 $t_{1}$ is $x$, and $x$ does not occur in $t_{2}$

3.4.5 $t_{1}$ is not $x, x$ occurs in $t_{1}$, and it does not occur in $t_{2}$.

Symmetrical cases to these from (3.4.1) - (3.4.5) are omitted in the above list. 
It is easy to check that in case (3.4.1), (3.4.2) $\mathrm{P}$ is constantly true when $\mathrm{x}$ gets any value from $|\underline{B}|$. Therefore we set in these cases $\mathrm{P}^{(\mathrm{x})}$ to be true.

In case (3.4.3) - (3.4.5) $P$ is constantly false for every value of $x$ in $|\underline{B}|$, and we set $P^{(x)}$ to be false.

The reader can easily check that (i) and (ii) are satisfied. Inductive step being obvious is omitted.

Before proceeding to the last auxiliary result let us observe that due to symmetry of construction of $\underline{A} \oplus \underline{B}$ Lemmas 3.3 and 3.4 remain true when $\ell_{1}$ is interchanged with $\ell_{2}$ and A with B.

\subsection{Lemma}

For every first order formula $P$ over the language of $\underline{A} \oplus \underline{B}$ there exist a nonnegative integer $n$, formulae $F_{1}, \ldots, F_{n}$ over $\mathcal{L}_{1} \cup\{A, B\}$, and formulae $G_{1}, \ldots, G_{n}$ over $\ell_{2} \cup\{A, B\}$ such that

$$
\underline{A} \oplus \underline{B} \vDash \prod_{i=1}^{n}\left(\left(F_{i}\right)_{A} \vee\left(G_{i}\right)_{B}\right)
$$

Proof: We prove the statement of Lemma 3.5 by induction on $P$.

Let $P$ be of the form $t_{1}=t_{2}$. Consider the following cases.

3.5.1 $t_{1}$ is a variable, say $x$. In $t_{2}$ there occur symbols from $\ell_{1}$, and $\ell_{2}$.

3.5.2 $t_{1}$ is over $\ell_{1}, t_{2}$ is over $\ell_{2}$, and both $t_{1}$ and $t_{2}$ are not variables.

3.5.3 $t_{1}$ is over $\ell_{1}$, in $t_{2}$ there are symbols from $\ell_{1}$ and $\ell_{2}$.

3.5.4 $t_{1}$ and $t_{2}$ are over $L_{i} \cup\{A, B, \perp\}$, where $i=1$ or $i=2$.

Cases symmetric to these listed above are omitted. Observe that if $t_{1}$ and $t_{2}$ satisfy (3.5.1), then $\underline{A} \oplus \underline{B} \vDash X=\perp \leftrightarrow P . \quad$ For (3.5.2) we have $\underline{A} \oplus \underline{B} \vDash P$

Suppose $P$ satisfies (3.5.3), let $X_{i}$ for $i=1.2$ be the set of all variables which occur in $t_{i}$. If $X_{1}=\varnothing$ or $X_{2}=\varnothing$, then obviously $\underline{A} \oplus \underline{B} \vDash \neg P$, otherwise it is easy to see that $\underline{A} \oplus \underline{B} \vDash P \leftrightarrow$ $\left[\left({ }_{x_{i} \in x_{1}}^{W} x_{i}=\perp\right) \wedge\left(W_{x_{i} \in X_{2}}^{W} x_{i}=\perp\right)\right]$. 
In case (3.5.4) obviously $\underline{A} \oplus \underline{B} \vDash P \leftrightarrow t_{1}=\perp$ holds.

Observe now that in cases (3.5.1) - (3.5.5) $\mathrm{P}$ is equivalent over $\underline{A} \oplus \underline{\mathrm{B}}$ to a formula over $\mathcal{L}_{\mathrm{i}}$ $\cup\{A, B, \perp\}$, where $i=1$ or $i=2$. Using Lemma 3.3 we obtain a desired decomposition of $P$.

Other cases of $\mathrm{P}$ being an atomic formula are dealt similarly and we omit them.

The only nontrivial case in the inductive step is $P$ being of the form $\forall \times Q$, where $\underline{A} \oplus \underline{B} \vDash$ $Q \leftrightarrow M_{i=1}^{n}\left(\left(F_{i}\right)_{A} \vee\left(G_{i}\right)_{B}\right)$ for certain $F_{i}$ 's over $\ell_{1} \cup\{A, B\}$ and $G_{i}$ 's over $\ell_{2} \cup\{A, B\}$.

Since $\quad \underline{A} \oplus \underline{B} \vDash(\forall x P) \leftrightarrow \sum_{i=1}^{n} \forall x\left(\left(F_{i}\right)_{A} \vee\left(G_{i}\right)_{B}\right)$,

it is enough to show a transformation of every formula $\forall x\left(\left(F_{i}\right)_{A} \vee\left(G_{i}\right)_{B}\right)$ for $i=1, \ldots, n$ into a formula of desired form. First we observe that such a formula is equivalent over $\underline{A} \oplus \underline{B}$ to the disjunction of these formulae

$$
\begin{array}{ll}
3.5 .5 & \left(\left(F_{i}\right)_{A}(\perp / x) \vee\left(G_{i}\right)_{A}(\perp / x)\right) \wedge \\
3.5 .6 & \forall x\left[A(x) \Rightarrow\left(\left(F_{i}\right)_{A} \vee\left(G_{i}\right)_{B}\right)\right] \wedge \\
\text { 3.5.7 } & \forall x\left[B(x) \Rightarrow\left(\left(F_{i}\right)_{A} \vee\left(G_{i}\right)_{B}\right)\right]
\end{array}
$$

Using Lemma 3.3 we transform formula (3.5.6) into an equivalent one which has a desired form. transformations of (3.5.7) and (3.5.8) are similar and we present here only a transformation of (3.5.7).

By Lemma $3.4(3.5 .7)$ is equivalent over $\underline{A} \oplus \underline{\oplus}$ to

$$
\forall x\left[A(x) \Rightarrow\left(\left(F_{i}\right)_{A} \vee\left(G_{i}^{(x)}\right)_{B}\right)\right]
$$

Since in $\left(G_{i}{ }^{(x)}\right)_{B} \quad x$ does not occur free, (3.5.9) is equivalent over $\underline{A} \oplus \underline{B}$ to $\left(\forall x F_{i}\right)_{A} \vee\left(G_{i}{ }^{(x)}\right)_{B}$.

This completes the proof of Lemma 3.5.

\subsection{Proof of Proposition 3.2}

Let $P$ be a first order formula over the language of $\underline{A} \oplus \underline{B}$. By Lemma 3.5 it is equivalent over $\underline{A} \oplus \underline{B}$ to $\bigwedge_{i=1}^{n}\left[\left(F_{i}\right)_{A} \vee\left(G_{i}\right)_{B}\right]$, where $F_{i}$ 's are over $\ell_{1} \cup\{A, B\}$ and $G_{i}$ 's are over $\ell_{2} \cup$ $\{A, B\}$. 
Let $X$ be the set of all variables which occur free in $P$. By Lemma 3.4 for every $1 \leq i \leq$ $n, A(X) \wedge\left(G_{i}\right)_{B}$ has a constant true value over $\underline{A} \oplus \underline{B}$ (i.e. it is equivalent over $\underline{A} \oplus \underline{B}$ to a sentence. Let $\epsilon_{i}$ be true if $\underline{A} \oplus \underline{B} \vDash A(X) \wedge\left(G_{i}\right)_{B}$, and false otherwise obviously.

3.6.1 $\underline{A} \oplus \underline{B} \vDash(A(X) \wedge P) \quad \leftrightarrow \quad A(X) \wedge M_{i=1}^{n}\left(\left(F_{i}\right)_{A} \vee \epsilon_{i}\right)$

Let $F_{i}{ }^{*}$ be obtained from $F_{i}$ by replacing in $F_{i}$ every subformula of the form $A(t)$ by true, and every subformula of the form $B(t)$ by false. It follows easily from (3.6.1) that we can take as the sought $Q: \bigwedge_{i=1}^{n}\left(F_{i}^{*} \vee \epsilon_{i}\right)$. This completes the proof of proposition 3.2.

\section{References}

1. Bergstra, J.A., \& J.V. Tucker, "Some natural structures which fail to possess a sound and decidable Hoare-like logic for their while-programs" (to appear in TCS. An earlier edition of this paper is registered at the Mathematical Centre as report IW 136/80).

2. Bergstra, J.A. \& J.F. Tucker, "Expressiveness and the completeness of Hoare's logic", Mathematical Centre Report IW 143/80.

3. Bergstra, J.A., \& J.V. Tucker, "Two theorems on the completeness of Hoare's Logic", Mathematical Centre Report IW?/81.

4. Cook, S.A., "Soundness and completeness of an axiom system for program verification", SIAM J. Computing ? (1978) 70-90.

5. Harel, D., "First order dynamic logic", Lecture notes in Computer Science 68, Springer 1978.

6. Wand, M., "A new incompleteness result for Hoare's system", JACM 25(1978) 168-175. 